\title{
Ossicle vs. avulsion fracture: Cinematic rendering of MDCT data as an additional diagnostic key
}

\author{
Georg Hagleitner ${ }^{1 *}$ and Franz A Fellner ${ }^{1,2}$ \\ ${ }^{1}$ Central Radiology Institute, Kepler University Hospital, Medical Faculty of the Johannes Kepler University, Linz, Austria \\ ${ }^{2}$ Medical Faculty of the Friedrich-Alexander-University of Erlangen-Nürnberg, Erlangen, Germany
}

\section{Background}

The differentiation between variants within the normal range and pathologic findings is a frequent challenge to radiologists in the daily routine. In trauma patients for example, it may be difficult to differentiate an ossicle from an avulsion fracture, even in crosssectional images [1]. Ossicles are a frequent finding and usually require no further therapy. On the other hand, through their relation to tendons or ligaments, avulsion fractures are frequently considered as "complex fractures" and require conservative and - in some cases - surgical management [2-4]. Thus, the clear differentiation between ossicles and avulsion fractures, even in anatomically difficult regions, is an important aspect of the radiologist's report [5]. We present a case in which cinematic rendering of MDCT data was a helpful key in the differentiation between an ossicle and an avulsion fracture.

\section{Case report}

We report a 31-year-old male patient with recent trauma to the right knee. After initial clinical examination and plain radiograms of the knee an avulsion fracture of the intercondylar eminence was suspected, differential diagnosis ossicle (Figure 1).

Subsequently, the patient was referred to computed tomography (CT) examination for further evaluation. A non-contrast conventional

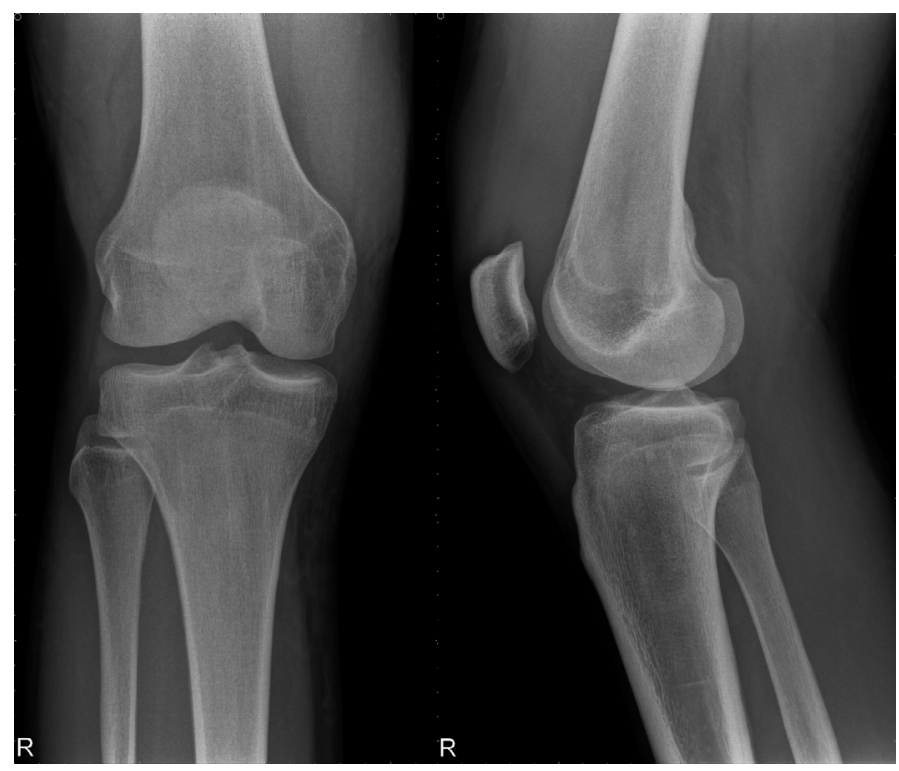

Figure 1. Conventional X-ray in ap (left) and lateral (right) view: Bone dense formation next to the intercondylar eminence, suspicious of an avulsion fracture. multi-detector computed tomography (MDCT) with axial slicing was carried out. Multiplanar reformations (MPR) were acquired in coronal and sagittal orientation. In postprocessing, a 3D-cinematic rendered overview for the whole volume and a separate reconstruction of the tibial head were conducted. Conventional MDCT images confirmed the bone dense formation close to the intercondylar eminence, suspicious of an ossicle. No other bone lesions were detected (Figure 2).

Additionally, 3D cinematic rendered images clearly confirmed that the bone dense structure was an ossicle in the intercondylar eminence (Figure 3).

\section{Discussion}

Especially in difficult anatomic regions it might be hard to differentiate between variations within the normal range and pathological findings even in MDCT images [5]. Regarding the presented case, radiological reports are frequently inconclusive in the differentiation between ossicles and avulsion fractures. This might lead to further, cost-intensive imaging and may delay immediate therapy[1,3,5].

Ossicles usually are sharply demarcated and show a sclerosed border. They are a frequent finding in articular and periarticular regions and appear in every age group. Normally, ossicles require no further therapy. Avulsion fractures on the other hand have a relation to the

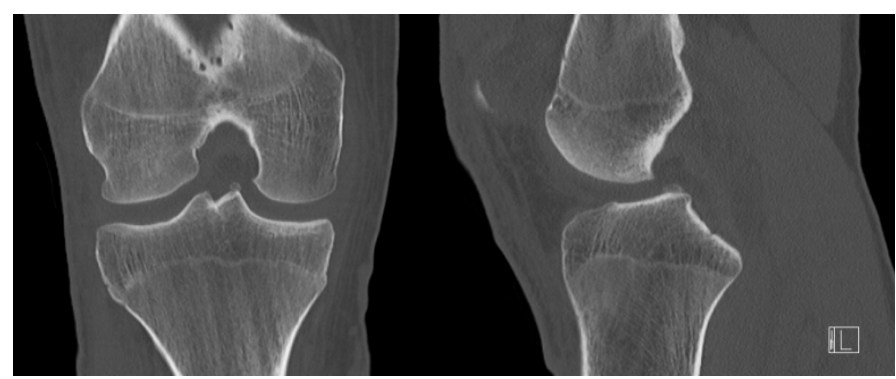

Figure 2. Coronar (left) and sagittal (right) MPR images from MDCT show an ossicle in the intercondylar eminence. There is no avulsion fracture.

Correspondence to: Georg Hagleitner, Central Radiology Institute, Kepler University Hospital, Medical Faculty of the Johannes Kepler University, Linz, Austria, E-mail: georg.hagleitner@kepleruniklinikum.at

Key words: ossicle, avulsion fracture, knee, MDCT, cinematic rendering, $3 D$ imaging

Received: October 03, 2017; Accepted: October 17, 2017; Published: October 20, 2017 


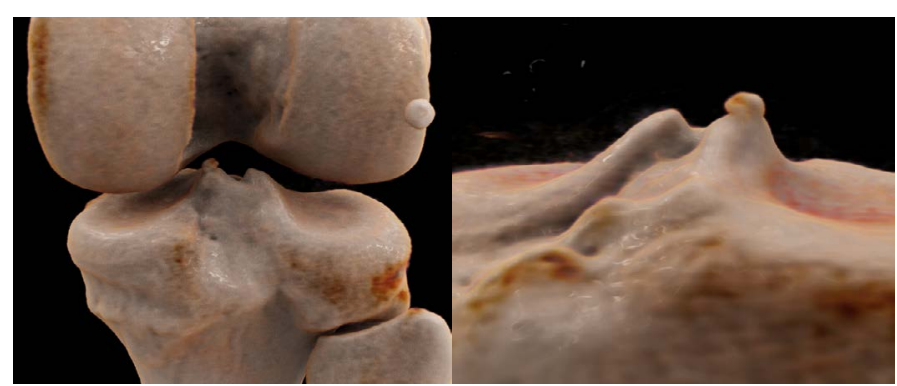

Figure 3. 3D-cinematic rendered images, posterior view of the knee (left) and ventromedial view of the tibial plateau (right) clearly visualize an ossicle in the intercondylar eminence.

origin or onset of a tendon or ligament, thus they might appear intraor periarticular regions as well. On conventional MDCT images they usually (partially) miss a sclerosed border as a sign of fracture $[1,3,4]$. However, even in new generation MSCTs with high resolution, the differentiation can be difficult or inconclusive. In our case, 3D-cinematic rendering was very helpful in addition to the MDCT source and MPR images concerning the differentiation of these diagnoses. It was a useful diagnostic addition to the conventional MDCT images by perfectly illustrating the difficult anatomy and its variants.

\section{Conclusion}

In conclusion, 3D-cinematic rendered images can be a helpful adjunct in cases with inconclusive findings and/or difficult anatomic circumstances and play a role for better illustration in interdisciplinary conferences [6,7].

\section{Disclosure}

No conflicts of interest. No grants or financial supports.

\section{References}

1. Guvenc Z, Ilaslan H, Sundaram M (2015) Medial Meniscal Ossicle. Orthopedics 10 457-458.

2. Atsumi S, Arai Y, Nakagawa S, Inoue H, Ikoma K, et al. (2016) A case of nonunion avulsion fracture of the anterior tibial eminence. Case Rep Orthop 2016: 1-5. [Crossref]

3. Devgan A, Mukhopadhyay R, Singh A, Gogna P, Singla R, Magu NK (2014) Ossicle in anterior cruciate ligament: A rare occurrence. Case Rep Orthop 2014: 1-3.

4. Hooper PO, Silko C, Malcolm TL, Farrow LD (2017) Management of posterior cruciate ligament tibial avulsion injuries: A systematic review. Am J Sports Med. [Crossref]

5. Doornberg JN, Rademakers MV, van den Bekerom MP, Kerkhoffs GM, Ahn J, et al. (2011) Two-dimensional and three-dimensional computed tomography for the classification and characterisation of tibial plateau fractures. Injury2: 1416-1425. [Crossref]

6. Rowe SP, Fritz J, Fishman EK (2017) CT evaluation of musculoskeletal trauma: initia experience with cinematic rendering. Emerg Radiol. [Crossref]

7. Fellner FA (2016) Introducing cinematic rendering: A novel technique for postprocessing medical imaging data. J Biomed Sci Eng 09: 170-175.

Copyright: (C2017 Hagleitner G. This is an open-access article distributed under the terms of the Creative Commons Attribution License, which permits unrestricted use, distribution, and reproduction in any medium, provided the original author and source are credited. 